\title{
New spectral bounds on the chromatic number encompassing all eigenvalues of the adjacency matrix
}

\author{
Pawel Wocjan \\ Mathematics Department \& Center for Theoretical Physics \\ Massachusetts Institute of Technology \\ Cambridge, MA, U.S.A. \\ Department of Electrical Engineering and Computer Science \\ University of Central Florida \\ Orlando, FL, U.S.A \\ wocjan@eecs.ucf .edu \\ Clive Elphick \\ clive.elphick@gmail.com
}

\begin{abstract}
Submitted: Sep 22, 2012; Accepted: Sep 4, 2013; Published: Sep 13, 2013
Mathematics Subject Classifications: 97K30, 97H60
\end{abstract}

\begin{abstract}
The purpose of this article is to improve existing lower bounds on the chromatic number $\chi$. Let $\mu_{1}, \ldots, \mu_{n}$ be the eigenvalues of the adjacency matrix sorted in non-increasing order.

First, we prove the lower bound $\chi \geqslant 1+\max _{m}\left\{\sum_{i=1}^{m} \mu_{i} /-\sum_{i=1}^{m} \mu_{n-i+1}\right\}$ for $m=1, \ldots, n-1$. This generalizes the Hoffman lower bound which only involves the maximum and minimum eigenvalues, i.e., the case $m=1$. We provide several examples for which the new bound exceeds the Hoffman lower bound.

Second, we conjecture the lower bound $\chi \geqslant 1+s^{+} / s^{-}$, where $s^{+}$and $s^{-}$are the sums of the squares of positive and negative eigenvalues, respectively. To corroborate this conjecture, we prove the bound $\chi \geqslant s^{+} / s^{-}$. We show that the conjectured lower bound is true for several families of graphs. We also performed various searches for a counter-example, but none was found.

Our proofs rely on a new technique of considering a family of conjugates of the adjacency matrix, which add to the zero matrix, and use majorization of spectra of self-adjoint matrices.

We also show that the above bounds are actually lower bounds on the normalized orthogonal rank of a graph, which is always less than or equal to the chromatic number. The normalized orthogonal rank is the minimum dimension making it
\end{abstract}


possible to assign vectors with entries of modulus one to the vertices such that two such vectors are orthogonal if the corresponding vertices are connected.

All these bounds are also valid when we replace the adjacency matrix $A$ by $W * A$ where $W$ is an arbitrary self-adjoint matrix and $*$ denotes the Schur product, that is, entrywise product of $W$ and $A$.

Keywords: chromatic number, spectral bounds, majorization

\section{Introduction}

Determining, and even approximating, the chromatic number of a graph is NP-hard [13, 20], so attention has focussed on upper and lower bounds. Upper bounds involve finding a coloring but lower bounds are more subtle, because they are based on demonstrating that no coloring exists for a given number of colors. The eigenvalues of a graph provide information about the whole graph whereas degrees provide information about individual vertices. As a result the best known lower bounds for the chromatic number are spectral [19], and in this paper we improve these bounds by incorporating all eigenvalues. We also conjecture a relationship between the sign of the eigenvalues and the chromatic number, which if true could lead to further developments in spectral graph theory.

Some of the ideas and techniques used in the proofs of these new bounds originated in the context of quantum information theory. We briefly explain below that our new technique of conjugating the adjacency matrix with unitary matrices to add to the zero matrix is an abstraction of a control-theoretic problem studied in quantum information theory [31].

Examples of matrices associated to graphs are the adjacency matrix, the Laplacian, and the signless Laplacian. In this paper, we focus on the adjacency matrix. Given an undirected graph $G$ with vertex set $V=\{1, \ldots, n\}$ and edge set $E$, the adjacency matrix of $G$ is a matrix $A=\left(a_{k \ell}\right)$ of size $n$ given by

$$
a_{k \ell}= \begin{cases}1 & \text { if }(k, \ell) \in E \\ 0 & \text { otherwise }\end{cases}
$$

The adjacency matrix $A$ is symmetric, and much is known about the spectra of such matrices. For instance, the eigenvalues of $A$ are real numbers, which we denote by $\mu_{1}, \ldots, \mu_{n}$, sorted in non-increasing order.

The Hoffman lower bound on the chromatic number [19]

$$
\chi \geqslant 1+\frac{\mu_{1}}{-\mu_{n}}
$$

is one of the best known results in spectral graph theory. There are several tests that a new lower bound for the chromatic number should satisfy to be of interest. These are that the bound:

- is exact for some class(es) of graphs; 
- exceeds the clique number for some graphs; and

- exceeds the Hoffman lower bound for the chromatic number for some graphs.

A different sort of test is how well it performs for random graphs.

We prove a new lower bound that generalizes the Hoffman lower bound and conjecture a new lower bound that satisfy the above tests.

Theorem 1. The chromatic number is bounded from below by

$$
\chi \geqslant 1+\max _{m=1, \ldots, n-1}\left\{\frac{\sum_{i=1}^{m} \mu_{i}}{-\sum_{i=1}^{m} \mu_{n-i+1}}\right\} .
$$

This bound reduces to the Hoffman bound when restricted to $m=1$.

To formulate our conjectured lower bound on the chromatic number, we need to introduce some further notation. The inertia of $A$ is the ordered triple $(\pi, \nu, \delta)$, where $\pi, \nu$ and $\delta$ are the numbers (counting multiplicities) of positive, negative and zero eigenvalues of $A$ respectively. Let

$$
s^{+}=\mu_{1}^{2}+\ldots+\mu_{\pi}^{2} .
$$

Let $m=|E|$ denote the number of edges. Since $\sum_{i=1}^{n} \mu_{i}^{2}=\operatorname{Tr}\left(A^{2}\right)=2 m$, it follows that

$$
s^{-}=\mu_{n-\nu+1}^{2}+\ldots+\mu_{n}^{2}=2 m-s^{+} .
$$

Conjecture 2. The chromatic number is bounded from below by

$$
\chi \geqslant 1+\frac{s^{+}}{s^{-}} .
$$

While we were not able to prove the above conjectured lower bound, we can prove the following bound.

Theorem 3. The chromatic number is bounded from below by

$$
\chi \geqslant \frac{s^{+}}{s^{-}} .
$$

It is possible to further improve the above generalized Hoffman bound and the above bound. Let $W$ be an arbitrary self-adjoint matrix. Denote by $\mu_{1}(W * A), \ldots, \mu_{n}(W * A)$ the eigenvalues of the Schur product $W * A$, ordered in non-increasing order.

Theorem 1 and the following more general result were first proved by Wocjan in the unpublished $\mathrm{PhD}$ thesis [31].

Theorem 4. The chromatic number is bounded from below by

$$
\chi \geqslant 1+\max _{W} \max _{m=1, \ldots, n-1}\left\{\frac{\sum_{i=1}^{m} \mu_{i}(W * A)}{-\sum_{i=1}^{m} \mu_{n-i+1}(W * A)}\right\},
$$

where $W$ ranges over all self-adjoint matrices and $\mu_{1}(W * A), \ldots, \mu_{n}(W * A)$ are the eigenvalues of the Schur product $W * A$ sorted in non-increasing order. 
We recover Theorem 1 by restricting to the case where $W$ is the all-one-matrix $J$. We also point out that Theorem 4 can be seen as a generalization of Lovász's result [24] in which $W$ ranges over all symmetric matrices and $m=1$.

The following lower bound on the chromatic number due to Barnes [2] can also be understood as a special case of Theorem 4. The chromatic number is bounded from below by

$$
\chi \geqslant 1+\max _{D}\left\{\mu_{1}\left(D^{-\frac{1}{2}} A D^{-\frac{1}{2}}\right)\right\},
$$

where $\mu_{1}\left(D^{-\frac{1}{2}} A D^{-\frac{1}{2}}\right)$ denotes the maximum eigenvalue of the weighted adjacency matrix $D^{-\frac{1}{2}} A D^{-\frac{1}{2}}$ and $D=\operatorname{diag}\left(d_{1}, \ldots, d_{n}\right)$ ranges over all diagonal matrices such that $A+D$ is positive semidefinite. (The condition $A+D$ is positive semidefinite implies that all $d_{1}, \ldots, d_{n}$ are necessarily positive so that we can form the matrix $D^{-\frac{1}{2}}$. Moreover, the maximization process over such $D$ can be solved efficiently with linear programming.

The Hoffman bound occurs as a special case of this theorem by setting $D=-\mu_{n} I$. The lower bound in eq. (1) follows as a special case of Theorem 4, which is seen by setting $W=\left(w_{i j}\right)=\left(d_{i}^{-\frac{1}{2}} d_{j}^{-\frac{1}{2}}\right)$. An important aspect of this result is that when $W$ is restricted to have this special form and $m=1$, then the maximization process over such restricted $W$ can be solved efficiently with the help of linear programming. It is not clear how to obtain the best possible $W$ and $m$ when no restrictions are placed on $W$ and $m$.

Theorem 5. The chromatic number is bounded from below by

$$
\chi \geqslant \max _{W}\left\{\frac{s^{+}(W * A)}{s^{-}(W * A)}\right\}
$$

where $W$ ranges over all self-adjoint matrices, $s^{+}(W * A)$ is the sum of the squares of the positive eigenvalues $\mu_{1}(W * A), \ldots, \mu_{\pi}(W * A)$ and $s^{-}(W * A)$ the sum of the squares of the negative eigenvalues $\mu_{n-\nu+1}(W * A), \ldots, \mu_{n}(W * A)$ of the Schur product $W * A$.

The paper is organized as follows. In Section 2, we prove the generalized Hoffman bound (Theorems 1 and 4). First, we present a new technique of conjugating the adjacency matrix with certain diagonal unitary matrices that are constructed from Fourier matrices, which add to the zero matrix. Second, we review some basic results of majorization theory of spectra of self-adjoint matrices. Third, we apply these majorization results to a matrix equality obtained by the conjugation technique to derive the generalized Hoffman bound. In Section 3, we prove Theorem 5. In Section 4, we present evidence for Conjecture 2. In Section 5, we discuss the performance of the generalized Hoffman bound and Conjecture 2.

\section{Generalized Hoffman bound}

\subsection{Conjugation of the adjacency matrix}

Recall that $G$ is colorable with $c$ colors if there exists a map $\Phi: V \rightarrow C=\{1, \ldots, c\}$ such that $a_{k \ell}=1$ implies $\Phi(k) \neq \Phi(\ell)$ for all $k, \ell \in V$. In words, the graph can be colored with 
$c$ colors if it is possible to assign at most $c$ different colors to its vertices such that any two adjacent vertices receive different colors. The chromatic number $\chi$ is the minimum number of colors required to color the graph.

The following new technique of constructing a family of conjugates of the adjacency matrix which add to the zero matrix is at the heart of our new bounds. Here $\dagger$ denotes the operation of taking the transpose of a matrix and changing its entries to their complex conjugates.

Theorem 6. Assume that there exists a coloring of $G$ with c colors. Then, there exist $c$ diagonal unitary matrices $U_{1}, \ldots, U_{c}$ whose entries are cth roots of unity such that

$$
\sum_{s=1}^{c} U_{s}(W * A) U_{s}^{\dagger}=0,
$$

where $W$ is an arbitrary self-adjoint matrix and $W * A$ denotes the entry-wise product of $W$ and $A$.

Moreover, this equality remains valid if we replace these diagonal unitary matrices $U_{s}$ by their inverses $U_{s}^{\dagger}$ for $s=1, \ldots, c$.

We also formulate the following simple corollary since it leads to the proof of Theorem 4 and Theorem 5.

Corollary 7. Assume that there exists a coloring of $G$ with $c$ colors. Then, there exist $c-1$ diagonal unitary matrices $U_{1}, \ldots, U_{c-1}$ whose entries are cth roots of unity such that

$$
\sum_{s=1}^{c-1} U_{s}(-W * A) U_{s}^{\dagger}=W * A .
$$

Moreover, this equality remains valid if we replace these diagonal unitary matrices $U_{s}$ by their inverses $U_{s}^{\dagger}$ for $s=1, \ldots, c-1$.

Remark 8. We point out that the "sign reversal map" $-W * A \mapsto W * A$ described in the corollary above is an abstraction of a problem in quantum control theory $[22,31]$. A closed quantum system evolves according to $\psi(t)=e^{-i H t} \psi(0)$, where the self-adjoint operator $H$ is the system Hamiltonian and $\psi(t)$ is a vector specifying the configuration of the quantum system at time $t$.

The quantum system can be made to evolve backwards in time by interspersing its natural time evolution $e^{-i H t}$ with certain external control operations which correspond to unitary operations. This task amounts to effectively changing the Hamiltonian $H$ to $-H$.

The $k$ th vertex of the graph corresponds to the $k$ th subsystem and the presence of an edge between vertices $k$ and $\ell$ indicates that $H$ couples the corresponding subsystems. The unitary matrix $U_{s}$ corresponds to an external control operation and $s$ indicates the step of the overall control sequence.

It can be shown that the cost of inverting the time evolution is bounded from above by a quantity related to the chromatic number of the graph characterizing the coupling 
topology of $H$ and is bounded from below by an expression involving eigenvalues of $H$. These results correspond to the generalized Hoffman's lower bound on the chromatic number.

Before proving the theorem and corollary we need to define Fourier matrices. For each $c$, the matrix

$$
F_{c}=\left(\begin{array}{cccc}
\zeta^{1 \cdot 1} & \zeta^{1 \cdot 2} & \ldots & \zeta^{1 \cdot c} \\
\zeta^{2 \cdot 1} & \zeta^{2 \cdot 2} & \ldots & \zeta^{2 \cdot c} \\
\vdots & \vdots & \ddots & \vdots \\
\zeta^{c \cdot 1} & \zeta^{c \cdot 2} & \ldots & \zeta^{c \cdot c}
\end{array}\right)
$$

where $\zeta$ is an arbitrary primitive $c$ th root of unity, is called a Fourier matrix. ${ }^{1}$ We have $F_{c} F_{c}^{\dagger}=c I$, that is, its rows are orthogonal vectors and $F_{c}^{\dagger} F=c I$, that is, its columns are orthogonal vectors. Observe that the last row of $F_{c}$ is equal to the all-one row vector and the last column of $F_{c}$ is equal to the all-one column vector.

Proof of Theorem 6. Let $\Phi: V=\{1, \ldots, n\} \rightarrow C=\{1, \ldots, c\}$ be a coloring of $G$. The entries of the $s$ th column of $F_{c}$ determine the entries of diagonal unitary matrix $U_{s}$. More precisely, the $k$ th diagonal entry of $U_{s}$, which corresponds to vertex $k$, is set to be $\Phi(k)$ th entry of the sth column of $F_{c}$. Therefore, vertices that have the same color select the same row of $F_{c}$ and vertices that have different colors select different rows.

For $s=1, \ldots, c$, we set

$$
U_{s}=\operatorname{diag}\left(\zeta^{\Phi(1) \cdot s}, \zeta^{\Phi(2) \cdot s}, \ldots, \zeta^{\Phi(n) \cdot s}\right)
$$

Observe that the multiplication of $W * A$ by the diagonal unitary matrix $U_{s}$ from the left corresponds to the multiplication of the $k$ th row of $W * A$ by the $k$ th diagonal entry of $U_{s}$ for $k=1, \ldots, n$. Similarly, the multiplication of $W * A$ by the diagonal unitary matrix $U_{s}^{\dagger}$ from the right corresponds to the multiplication of the $\ell$ th column of $W * A$ by the eth diagonal entry of $U_{s}^{\dagger}$ for $\ell=1, \ldots, n$.

Therefore, the $(k, \ell)$ th entry of the sum of the conjugates

$$
\sum_{s=1}^{c} U_{s}(W * A) U_{s}^{\dagger}
$$

is equal to

$$
\sum_{s=1}^{c} \zeta^{\Phi(k) \cdot s} w_{k \ell} a_{k \ell} \zeta^{-\Phi(\ell) \cdot s}
$$

for $k, \ell=1, \ldots, n$.

If $\Phi(k)=\Phi(\ell)$, then necessarily $a_{k \ell}=0$ because vertices that receive the same color under the coloring $\Phi$ cannot be adjacent. Consequently, the corresponding entry of the above sum is equal to 0 .

\footnotetext{
${ }^{1}$ In the literature, it is often customary to start the row and column indices at 0 instead of 1 . For our purposes, it is more convenient to choose 1 .
} 
If $\Phi(k) \neq \Phi(\ell)$, then $a_{k \ell}=1$ or $a_{k \ell}=0$. Even if $a_{k \ell}=1$ the corresponding entry of the sum is also equal to 0 since

$$
\sum_{s=1}^{c} \zeta^{(\Phi(k)-\Phi(\ell)) \cdot s}=0
$$

This equality means that the $\Phi(k)$ th and $\Phi(\ell)$ th rows of $F_{c}$ are orthogonal.

Observe that replacing the diagonal unitary matrices $U_{s}$ by their inverses $U_{s}^{\dagger}$ corresponds to replacing the Fourier matrix $F_{c}$ by its adjoint $F_{c}^{\dagger}$, which is a Fourier matrix with respect to the primitive $c$ th root of unity $\bar{\zeta}$.

Proof. (Corollary 7) This result follows immediately because $U_{c}=U_{c}^{\dagger}=I$ since the last row and the last column of $F_{c}$ are the all-one row vector and the all-one column vector, respectively.

To further illuminate the process in Theorem 6 , we now consider $\sum_{s=1}^{c} U_{s} X U_{s}^{\dagger}$ for arbitrary matrices $X$ and not just matrices of the special form $W * A$.

Remark 9. Let $\Phi: V \rightarrow C=\{1, \ldots c\}$ be a coloring and $U_{1}, \ldots, U_{c}$ the corresponding diagonal unitary matrices constructed as in the proof of Theorem 6 . For $b=1, \ldots, c$, set

$$
P_{b}=\sum_{k \in \Phi^{-1}(b)} e_{k}^{\dagger} e_{k}
$$

where $e_{1}=(1,0, \ldots, 0)^{T}, \ldots, e_{n}=(0, \ldots, 0,1)^{T}$ are the standard basis vectors of $\mathbb{C}^{n}$. In words, $P_{b}$ is the orthogonal projector onto the subspace spanned by $e_{k}$ for which the corresponding vertex $k$ receives the color $b$ under the coloring $\Phi$.

For an arbitrary matrix $X$, we have

$$
\sum_{s=1}^{c} U_{s} X U_{s}^{\dagger}=c \sum_{b=1}^{c} P_{b} X P_{b} .
$$

The operation taking $X$ to $\mathcal{P}(X):=\sum_{b=1}^{c} P_{b} X P_{b}$ is known in the literature as pinching [3, Problem II.5.5].

\subsection{Majorization of spectra of self-adjoint operators}

We recall some basic definitions and results in majorization. We refer the reader to [3, Chapters II and III]. Let $x=\left(x_{1}, \ldots, x_{n}\right)$ be an element of $\mathbb{R}^{n}$. Let $x^{\downarrow}$ be the vector obtained by rearranging the coordinates of $x$ in the non-increasing order. Thus, if $x^{\downarrow}=$ $\left(x_{1}^{\downarrow}, \ldots, x_{n}^{\downarrow}\right)$, then $x_{1}^{\downarrow} \geqslant \ldots \geqslant x_{n}^{\downarrow}$.

Let $x, y \in \mathbb{R}^{n}$. We say that $x$ is majorized by $y$, in symbols $x \prec y$, if

$$
\sum_{i=1}^{m} x_{i}^{\downarrow} \leqslant \sum_{i=1}^{m} y_{i}^{\downarrow}
$$


for $m=1, \ldots, n-1$ and

$$
\sum_{i=1}^{n} x_{i}^{\downarrow}=\sum_{i=1}^{n} y_{i}^{\downarrow} .
$$

Let $A$ be a self-adjoint operator acting on $\mathbb{C}^{n}$ and $\mu(A)$ denote the vector in $\mathbb{R}^{n}$ whose coordinates are the eigenvalues of $A$ specified in any order.

Let $A, B$ be two arbitrary self-adjoint operators. Then, [3, Corollary III.4.2] shows that

$$
\mu^{\downarrow}(A+B) \prec \mu^{\downarrow}(A)+\mu^{\downarrow}(B) .
$$

\subsection{Proof of the generalized Hoffman bound}

Proof of Theorem 4. Consider Corollary 7 and the matrix equality

$$
\sum_{s=1}^{c-1} U_{s}(-W * A) U_{s}^{\dagger}=W * A .
$$

Using the result in eq. (5) and induction on $c$, we see that

$$
\mu^{\downarrow}(W * A) \prec \sum_{s=1}^{c-1} \mu^{\downarrow}\left(U_{s}(-W * A) U_{s}^{\dagger}\right) .
$$

Since conjugation of $-W * A$ by the unitary matrices $U_{s}$ leaves the spectrum invariant, we obtain

$$
\mu^{\downarrow}(W * A) \prec(c-1) \mu^{\downarrow}(-W * A) .
$$

Note that $\mu^{\downarrow}(-W * A)=\left(-\mu_{n}(W * A), \ldots,-\mu_{1}(W * A)\right)$. The result now follows by using the condition in eq. (4) for $m=1, \ldots, n-1$, dividing both sides by $-\sum_{i=1}^{m} \mu_{n-i+1}(W * A)$, and adding 1 to both sides.

\subsection{Relation to orthogonal representations}

We now strengthen the result of Theorem 6 . To do this, we need to introduce orthogonal representations of graphs as studied in $[8,17]$, which occur in the study of the quantum chromatic number. A $d$-dimensional orthogonal representation of $G$ is a map $\Psi: V \rightarrow \mathbb{C}^{d}$, mapping vertices to $d$-dimensional column vectors such that $a_{k \ell}=1$ implies $\Psi(k)^{\dagger} \Psi(\ell)=0$ for $k, \ell \in V$. The orthogonal rank of $G$, denoted by $\xi$, is the minimum $d$ such that there exists an orthogonal representation of $G$ in $\mathbb{C}^{d}$. Furthermore, let $\xi^{\prime}$ be the smallest $d$ such that $G$ has an orthogonal representation in the vector space $\mathbb{C}^{d}$ with the added restriction that the entries of each vector must have modulus one [8]. We refer to these representations as normalized orthogonal representations and to $\xi^{\prime}$ as the normalized orthogonal rank.

To see that $\xi^{\prime} \leqslant \chi$ holds, we color the vertices with $\chi$ colors according to the coloring $\Phi$ and assign the $\Phi(k)$ th column of $F_{\chi}$ to vertex $k$ for $k=1, \ldots, n$.

We now show there always exist $\xi^{\prime}$ diagonal unitary matrices $U_{1}, \ldots, U_{\xi^{\prime}}$ such that $\sum_{s=1}^{\xi^{\prime}} U_{s} A U_{s}^{\dagger}=0$, providing a strengthening of Theorem 6 . It therefore follows that the 
lower bounds on the chromatic number proved in this paper are in fact lower bounds on the normalized orthogonal rank.

Lemma 10. Let $U_{1}, \ldots, U_{d}$ be a collection of diagonal unitary matrices of size $n$ and use the notation

$$
U_{s}=\operatorname{diag}\left(u_{1, s}, \ldots, u_{n, s}\right) \quad \text { for } s=1, \ldots, d
$$

to denote the diagonal entries of the matrices $U_{s}$.

Then, we have

$$
\sum_{s=1}^{d} U_{s}^{\dagger} U_{s}=I \quad \text { and } \quad \sum_{s=1}^{d} U_{s} A U_{s}^{\dagger}=0
$$

if and only if the vectors

$$
\Psi_{k}=\left(u_{k, 1}, \ldots, u_{k, d}\right)^{T} \quad \text { for } k=1, \ldots, n
$$

form a normalized orthogonal representation of the graph with adjacency matrix $A$.

Proof. The entry on the left hand side of (7) in the $k$ th row and $\ell$ th column is equal to

$$
\sum_{s=1}^{d} \bar{u}_{k, s} u_{\ell, s} a_{k, \ell}=\Psi_{k}^{\dagger} \Psi_{\ell} a_{k, \ell},
$$

where equality follows from the definition of the vectors $\Psi_{k}$.

From [14], we see that for a Hadamard graph $G_{N}, \chi\left(G_{N}\right)>N$ whenever $N=4 m$ with $m>2$. In our current notation this says that almost all Hadamard graphs with $N=4 m$ have $\xi^{\prime}<\chi$, since trivially $\xi^{\prime}\left(G_{N}\right) \leqslant N$. A Hadamard graph $G_{N}=(V, E)$ is the graph with vertex set $V=\{0,1\}^{N}$ and edge set $E=\left\{(u, v) \in V \times V \mid d_{H}(u, v)=N / 2\right\}$, where $d_{H}$ is the Hamming distance. For each $k=\left(k_{1}, \ldots, k_{N}\right) \in V$, define an orthogonal representation by setting $\Psi(k)=\left((-1)^{k_{1}}, \ldots,(-1)^{k_{N}}\right)$. For any pair of adjacent vertices $k$ and $\ell$, we have $\Psi(k)^{\dagger} \Psi(\ell)=\sum_{s=1}^{N}(-1)^{k_{s}+\ell_{s}}=0$. This shows $\xi^{\prime}\left(G_{N}\right) \leqslant N$. The result [14, Theorem 7.1] states that $\chi\left(G_{N}\right)=N$ if and only if $N=2^{m}$ with $m \leqslant 3$, implying that $\chi\left(G_{12}\right)>12 \geqslant \xi^{\prime}\left(G_{12}\right)$.

Hadamard graphs are the only known graphs whose normalized orthogonal rank $\xi^{\prime}$ is strictly less than their chromatic number $\chi$. It would be interesting to find new families of graphs satisfying this strict inequality.

The above discussion shows that the generalized Hoffman bound (Theorems 1 and 4) and the new bound (Theorems 3 and 5) are both lower bounds on the normalized orthogonal rank $\xi^{\prime}$, which is always less than or equal to the chromatic number $\chi$. This is similar to Bilu's result that Hoffman's bound is a lower bound on the vector chromatic number [4], which in turn is bounded from above by the Lovász $\vartheta$ number of the complementary graph [23, Theorem 8.1]. We do not know the relationship between the vector chromatic number and the normalized orthogonal rank. The problem is that the vector chromatic number is defined using vectors with real entries, whereas the orthogonal rank is defined using vectors with complex entries. 


\section{The bound $\chi \geqslant s^{+} / s^{-}$}

Proof of Theorem 3. Let $A$ be an arbitrary self-adjoint matrix and $U$ an arbitrary unitary matrix, both acting on $\mathbb{C}^{n}$. Let

$$
A=\sum_{i=1}^{n} \mu_{i} v_{i} v_{i}^{\dagger}
$$

be the spectral resolution of $A$, that is, $\mu_{1}, \ldots, \mu_{n}$ are the eigenvalues of $A$ and $v_{1}, \ldots, v_{n}$ the corresponding row eigenvectors of unit length. We order the eigenvalues of $A$ in nonincreasing order, that is, $\mu_{1} \geqslant \mu_{2} \geqslant \ldots \geqslant \mu_{n}$. The eigenvectors $v_{i}$ can always be chosen so that they form an orthonormal basis of $\mathbb{C}^{n}$.

Let $\pi$ denote the number of positive eigenvalues of $A$ and set

$$
s^{+}=\sum_{i=1}^{\pi} \mu_{i}^{2}, \quad \text { and } \quad s^{-}=\sum_{i=n-\nu+1}^{n} \mu_{i}^{2} .
$$

Write $A=B-C$ where $B=\sum_{i=1}^{\pi} \mu_{i} v_{i} v_{i}^{\dagger}$ and $C=\sum_{i=n-\nu+1}^{n}\left(-\mu_{i}\right) v_{i} v_{i}^{\dagger}$. Note that $B$ and $C$ are both positive semidefinite, and $s^{+}=\operatorname{Tr}\left(B^{2}\right)$ and $s^{-}=\operatorname{Tr}\left(C^{2}\right)$.

Recall that a proper coloring (and also a normalized orthogonal representation) make it possible to find $c$ diagonal unitary matrices $U_{1}, \ldots, U_{c}$ such that $\sum_{i=1}^{c} U_{s} A U_{s}^{\dagger}=0$ and $\sum_{i=1}^{c} U_{s}^{\dagger} A U_{s}=0$. We obtain

$$
B=\sum_{s=1}^{c} U_{s} C U_{s}^{\dagger}-\sum_{s=1}^{c-1} U_{s} B U_{s}^{\dagger}
$$

Multiply both sides of the above equation by $B$ and take the trace of both sides:

$$
s^{+}=\operatorname{Tr}\left(B^{2}\right)=\operatorname{Tr}\left(\sum_{s=1}^{c} U_{s} C U_{s}^{\dagger} B\right)-\operatorname{Tr}\left(\sum_{s=1}^{c-1} U_{s} B U_{s}^{\dagger} B\right) .
$$

We employ the cyclic property of the trace to move $U_{s}$ from left to right, the identity $\sum_{i=1}^{c} U_{s}^{\dagger} A U_{s}=0$, and the cyclic property to move $U_{s}$ from right to left to establish

$$
\operatorname{Tr}\left(\sum_{s=1}^{c} U_{s} C U_{s}^{\dagger} B\right)=\operatorname{Tr}\left(\sum_{s=1}^{c} U_{s} C U_{s}^{\dagger} C\right)
$$

Therefore

$$
s^{+}=\operatorname{Tr}\left(\sum_{s=1}^{c} U_{s} C U_{s}^{\dagger} C\right)-\operatorname{Tr}\left(\sum_{s=1}^{c-1} U_{s} B U_{s}^{\dagger} B\right) .
$$

Observe that $\operatorname{Tr}\left(U_{s} B U_{s}^{\dagger} B\right)$ and $\operatorname{Tr}\left(U_{s} C U_{s}^{\dagger} C\right)$ are both non-negative numbers. They have the form $\operatorname{Tr}\left(V P V^{\dagger} P\right)$ and this expression can be written as $\operatorname{Tr}\left(\sqrt{P} V \sqrt{P}(\sqrt{P} V \sqrt{P})^{\dagger}\right)=$ $\operatorname{Tr}\left(X X^{\dagger}\right) \geqslant 0$ where $X=\sqrt{P} V \sqrt{P}$. We can take the square root of $P$ because $P$ represents either $B$ or $C$, which are both positive semidefinite. 
We now use that $\operatorname{Tr}\left(V P V^{\dagger} P\right) \leqslant \operatorname{Tr}\left(P^{2}\right)$ holds for all unitary matrices $V$ and all hermitian matrices $P$, which follows as a special case from [29, Lemma 3.4], to establish

$$
s^{+} \leqslant \operatorname{Tr}\left(\sum_{s=1}^{c} U_{s} C U_{s}^{\dagger} C\right) \leqslant c \operatorname{Tr}\left(C^{2}\right)=c s^{-} .
$$

The proof of Theorem 5 follows that for Theorem 3, but with $A$ replaced by $W * A$.

\section{Evidence for the conjectured bound $\chi \geqslant 1+s^{+} / s^{-}$}

We start by describing how the conjectured lower bound is related to known results in spectral graph theory. We describe a hierarchy of lower bounds starting from the weakest and ending at the conjectured lower bound.

Myers and Liu [21] proved the following degree-based bound

$$
\omega \geqslant 1+\frac{2 m}{n^{2}-2 m}
$$

on the clique number $\omega$. In 1972, Cvetkovic [9] proved that

$$
\chi \geqslant 1+\frac{\mu_{1}}{n-\mu_{1}} .
$$

Wilf [30] proved that this bound is in fact a lower bound for the clique number and therefore implies the concise Turán theorem. In 1983, Edwards and Elphick proved that

$$
\chi \geqslant 1+\frac{\mu_{1}^{2}}{2 m-\mu_{1}^{2}}
$$

and conjectured that $\chi$ can be replaced by the clique number $\omega[11,12]$. Nikiforov proved this conjecture in [25] and generalized it by replacing $\mu_{1}^{2}$ by $\mu_{1}^{r}$ and $2 m$ with the number of $r$-walks in $G$ [26].

Bollobás and Nikiforov conjectured that

$$
\omega \geqslant 1+\frac{\mu_{1}^{2}+\mu_{2}^{2}}{2 m-\mu_{1}^{2}-\mu_{2}^{2}}
$$

for non-complete graphs [6]. This conjecture is exact for complete bipartite and complete regular $q$-partite graphs, since $\mu_{2}=0$ for these graphs.

Note that it is not possible to replace the chromatic number with the clique number in Conjecture 2 because, for example, the Coxeter graph provides a counter-example. Smith [28] has proved that $\mu_{2}>0$ for all connected graphs other than complete multipartite graphs, so Conjecture 2 is an improvement on the result due to Edwards and Elphick for all such graphs. 
We have not been able to prove Conjecture 2. In addition to proving the bound in the previous section, we are able to identify three additional types of evidence in support of the conjecture. These are as follows:

First, the conjecture is exact for several graph families, including all bipartite, complete and complete regular $q$-partite graphs. For example, any bipartite graph has a spectrum which is symmetric about zero. Therefore for all bipartite graphs: $s^{+}=s^{-}$and consequently $1+s^{+} / s^{-}=2$ when $\chi=2$. The proofs for complete and complete regular $q$-partite graphs are in the Appendix to this paper.

Secondly, we have proved the conjecture is correct for strongly regular, complete $q$ partite and Regular Two-graphs and for Kneser graphs $K G_{p, k}$ for $k \leqslant 4$. Outline proofs are in the Appendix to this paper.

Finally we have conducted various searches for a counter-example. The Wolfram Mathematica 8.0 function GraphData[n] lists named graphs on $n$ vertices. We have searched the thousands of such graphs with $n \leqslant 50$ and found no counter-examples to Conjecture 2. Wilf proved the well known upper bound that $\chi \leqslant 1+\mu_{1}$. Therefore if there exists a graph for which $s^{+} / s^{-}>\mu_{1}$, this would provide a counter-example to Conjecture 2. Godsil has tested this inequality against all 274,668 graphs on 9 vertices using Sage and found no counter-examples [15]. The conjecture performs particularly well for small, dense random graphs of the form $G_{n, p}$, where $n$ is the number of vertices and $p$ is the independent probability of each edge being present. We have therefore used the Wolfram function RandomGraph [n,p] to generate over 100 graphs with $n=10$ and $p=0.85$ or 0.9 and again found no counter-examples.

\section{Empirical performance of the bounds}

The best known lower bound for $\chi$ is the Hoffman bound. Unlike the Myers and Liu, Cvetkovic, and Edwards and Elphick bounds, the Hoffman bound is not a lower bound for the clique number $\omega$. We have therefore compared the performance of Conjecture 2 and Theorem 1 with the performance of the Hoffman bound. We have focused on the performance of Conjecture 2 rather than of Theorem $3\left(\chi \geqslant s^{+} / s^{-}\right)$because we regard Theorem 3 as interesting rather than useful. Theorem 3 is occasionally better than the Hoffman bound, but we have not been able to find a graph for which Theorem 3 exceeds the clique number. We hope that Theorem 3 will become a stepping stone to a proof of Conjecture 2, rather than a significant result in its own right. Theorem 1 is a generalization of the Hoffman bound and so can never be worse than the Hoffman bound. We have not performed the maximization over $W$ in Theorem 4 and Theorem 5 .

We have made comparisons using both named and random graphs in Wolfram Mathematica 8.0. The results are set out below.

\subsection{Named graphs}

The Wolfram function GraphData [n] generates parameters for named graphs on $n$ vertices. For example, there are 78 named graphs on 16 vertices, excluding the complete, 
empty and bipartite graphs. Tabulated below are the numbers of such named graphs on 16, 25 and 28 vertices and the percentages of these graphs for which Theorem 1 and Conjecture 2 exceed the Hoffman bound:

$\begin{array}{cccc}n & \text { named graphs } & \text { Theorem 1 } & \text { Conjecture 2 } \\ 16 & 78 & 15 \% & 22 \% \\ 25 & 30 & 7 \% & 13 \% \\ 28 & 27 & 15 \% & 19 \%\end{array}$

An example of a graph for which the new bounds perform well is Barbell(8), for which the Hoffman bound is 4.8 , Theorem 1 is 5.9 , Conjecture 2 is 7.3 and the chromatic number is 8.

Theorem 1 and Conjecture 2 tend to perform particularly well for graphs that are nearly disconnected.

\subsection{Random Graphs}

The Wolfram function RandomGraph [n,p] generates a random graph $G_{n, p}$ on $n$ vertices with each edge being present with independent probability $p$. Eigenvalues are found using the function Spectrum, provided the Wolfram package "Combinatorica" has been loaded. Theorem 1 almost never exceeds the Hoffman bound for random graphs, because for almost all random graphs $\mu_{1} \gg \mu_{2}$, and consequently generalizing over more eigenvalues than Hoffman does not improve the bound.

Tabulated below is the performance of Conjecture 2 against the Hoffman bound for each combination of $n=20$ and 50 and $p=0.5,0.7$ and 0.9 , in each case averaged over 15 graphs. We have included a comparison with the 1988 result due to Bollobás [5] that the chromatic number of almost every random graph $G_{n, p}$ is: $q=(1 / 2+o(1)) n / \log _{b}(n)$, where $b=1 /(1-p)$.

$\begin{array}{ccccc}n & p & \text { Hoffman Bound } & \text { Conjecture 2 } & \text { Bollobás } \\ 20 & 0.5 & 3.3 & 2.9 & 2.3 \\ 20 & 0.7 & 4.3 & 4.2 & 4.0 \\ 20 & 0.9 & 6.3 & 8.2 & 7.7 \\ 50 & 0.5 & 4.5 & 3.2 & 4.4 \\ 50 & 0.7 & 6.2 & 4.9 & 7.7 \\ 50 & 0.9 & 9.9 & 10.8 & 14.7\end{array}$

It can be seen that for $n=20$ both bounds exceed the Bollobás formula for varying levels of $p$, because for low levels of $n$ the $o(1)$ term is material. The main conclusion is that the performance of Conjecture 2 is strongly affected by graph density. For sparse graphs with $p=0.5$ the Hoffman bound is almost always better, irrespective of $n$. For dense graphs the position is more complex. With $p=0.9$, Conjecture 2 usually exceeds Hoffman for $n$ less than about 65 but is worse than Hoffman for $n$ greater than about 65 . The range of values of both bounds is fairly small. With a sample size of 15 , for $\mathrm{n}=50$ and $p=0.9$ the range of the Hoffman bound was 9.1-11.3 and the range for Conjecture 2 was 9.6-11.5. 


\section{Conclusions}

Most spectral bounds in graph theory involve a small number of eigenvalues. In this paper we have investigated two new lower bounds for the chromatic number, which involve all eigenvalues of the adjacency matrix. The principal open question raised by the paper is whether Conjecture 2 is true. If it is then it provides an unexpected relationship between the sign of the eigenvalues of the adjacency matrix of a graph and its chromatic number. This relationship does not apply to the clique number.

Underpinning our new bounds is Theorem 6, which is a new characterization of a $\chi$-chromatic graph.

Bilu has proved that the Hoffman bound is also a lower bound for the vector chromatic number [4]. We have shown that the generalized Hoffman bound is also a lower bound on the normalized orthogonal rank. The exact relationship between the vector chromatic number and the normalized orthogonal rank is an open question.

Finally we have not considered how to efficiently maximize over $W$ in Theorems 4 and 5 , but the paper by Barnes [2] provides an indication on how to proceed.

\section{Appendix - outline proofs of Conjecture 2 for various graph families}

\section{Strongly regular graphs}

A strongly $d$-regular graph has precisely three distinct eigenvalues, namely $\mu_{1}=d$ with multiplicity $1 ; \mu_{2}$ with multiplicity $f$; and $\mu_{n}$ with multiplicity $(n-f-1)$. The Hoffman lower bound for the chromatic number, $\chi$, of regular graphs is $\left(\mu_{n}-d\right) / \mu_{n}$. We are therefore seeking to prove that:

$$
s^{-}(G)=(n-f-1) \mu_{n}^{2} \geqslant \frac{n d \mu_{n}}{\left(\mu_{n}-d\right)}=\frac{2 m \mu_{n}}{\left(\mu_{n}-d\right)} \geqslant \frac{2 m}{\chi} .
$$

This simplifies to:

$$
(n-f-1) \mu_{n}\left(\mu_{n}-d\right) \geqslant n d .
$$

Since $\sum \mu_{i}=0$, it follows that $f=\left(d+(n-1) \mu_{n}\right) /\left(\mu_{n}-\mu_{2}\right)$. Inequality (13) therefore simplifies to the following, noting that $\left(\mu_{n}-\mu_{2}\right)<0$ :

$$
\left(-n \mu_{2}-d+\mu_{2}\right)\left(\mu_{n}^{2}-\mu_{n} d\right) \leqslant n d\left(\mu_{n}-\mu_{2}\right)
$$

Haemers [18] (and others) have noted that $\left(d-\mu_{2}\right)\left(d-\mu_{n}\right)=n\left(d+\mu_{2} \mu_{n}\right)$. After some algebra, inequality (14) therefore simplifies to:

$$
\mu_{2}\left(\mu_{n}+1\right) \leqslant 0
$$

However for all strongly regular graphs, $\mu_{2} \geqslant 0$ and $\mu_{n} \leqslant-1$. This completes the proof. 


\section{Complete $\chi$-partite graphs}

The complete regular $\chi$-partite graph with $s$ vertices in each color class has $\mu_{1}=(\chi-1) s$, $\mu_{2}=0$ and $2 m=\chi(\chi-1) s^{2}$. Therefore:

$$
s^{+}(G)=\mu_{1}^{2}=(\chi-1)^{2} s^{2}=2 m(\chi-1) / \chi,
$$

so Conjecture 2 is exact for complete regular $\chi$-partite graphs.

Irregular complete $\chi$-partite graphs also have $\mu_{2}=0$, so for these graphs:

$$
s^{+}(G)=\mu_{1}^{2} \leqslant 2 m(\chi-1) / \chi
$$

due to the results quoted in Section 4.

\section{Regular-Two graphs}

A Regular-Two graph is a graph with only two distinct eigenvalues, $\mu_{1}$ with multiplicity $v$ and $\mu_{n}$ with multiplicity $(n-v)$. Therefore $\mu_{1}=-(n-v) \mu_{n} / v, 2 m=\mu_{n}^{2}(n-v) n / v$ and $\chi \geqslant\left(\mu_{n}-\mu_{1}\right) / \mu_{n}=n / v$. Therefore:

$$
s^{-}(G)=(n-v) \mu_{n}^{2}=(n-v) \mu_{n}^{2} \frac{n}{v} \frac{v}{n}=2 m \frac{\mu_{n}}{\left(\mu_{n}-\mu_{1}\right)} \geqslant \frac{2 m}{\chi} .
$$

\section{Kneser graphs}

The Kneser graph $K G_{p, k}$ is the graph whose vertices correspond to the $k$-element subset of a set of $p$ elements, and where two vertices are joined if and only if the corresponding sets are disjoint. Properties of $K G_{p, k}$ include the following, with the eigenvalues due to Godsil and Royle [16]:

$$
\begin{aligned}
\chi & =p-2 k+2, \text { and hence } p \geqslant 2 k, \\
n & =\left(\begin{array}{l}
p \\
k
\end{array}\right), \\
2 m & =\left(\begin{array}{l}
p \\
k
\end{array}\right)\left(\begin{array}{c}
p-k \\
k
\end{array}\right)
\end{aligned}
$$

The eigenvalues are

$$
(-1)^{i}\left(\begin{array}{c}
p-k-i \\
k-i
\end{array}\right) \text { with multiplicity }\left(\begin{array}{c}
p \\
i
\end{array}\right)-\left(\begin{array}{c}
p \\
i-1
\end{array}\right) \text {, for } i=0,1,2, \ldots, k .
$$

For $k=1$ the Kneser graphs are the Complete graphs.

For $k=2$ there is only one distinct negative eigenvalue, with $i=1$. We are therefore seeking to prove that:

$$
s^{-}(G)=(p-1) \mu_{n}^{2}=(p-1)\left(\begin{array}{c}
p-3 \\
1
\end{array}\right)^{2} \geqslant \frac{1}{p-2}\left(\begin{array}{l}
p \\
2
\end{array}\right)\left(\begin{array}{c}
p-2 \\
2
\end{array}\right)=\frac{2 m}{\chi}
$$


This simplifies to $p \geqslant 4=2 k$ and we are done.

For $k=3$ there are two distinct positive eigenvalues, with $i=0$ and 2. The multiplicity when $i=2$ is $\left[\left(\begin{array}{l}p \\ 2\end{array}\right)-\left(\begin{array}{l}p \\ 1\end{array}\right)\right]$. We are therefore seeking to prove that:

$$
s^{+}(G)=\mu_{1}^{2}+\left[\left(\begin{array}{l}
p \\
2
\end{array}\right)-\left(\begin{array}{l}
p \\
1
\end{array}\right)\right] \mu_{2}^{2} \leqslant \frac{1}{p-4}\left(\begin{array}{l}
p \\
3
\end{array}\right)\left(\begin{array}{c}
p-3 \\
3
\end{array}\right)(p-5)=\frac{2 m(\chi-1)}{\chi} .
$$

Inserting the values for the eigenvalues and expanding the right side this becomes:

$$
\left(\begin{array}{c}
p-3 \\
3
\end{array}\right)^{2}+\frac{1}{2}\left(\begin{array}{c}
p-5 \\
1
\end{array}\right)^{2} p(p-3) \leqslant \frac{p(p-1)(p-2)(p-3)(p-5)^{2}}{36} .
$$

Simple algebra reduces this to:

$$
0 \leqslant p^{2}-7 p+6=(p-6)(p-1)
$$

which implies $p \geqslant 6=2 k$, and we are done.

A similar but longer proof works for $k=4$. We have not attempted a proof for $k>4$.

\section{Acknowledgements}

We would like to thank Dominik Janzing, Giannicola Scarpa and Simone Severini for helpful discussions. P.W. gratefully acknowledges the support from the National Science Foundation CAREER Award CCF-0746600. This work was supported in part by the National Science Foundation Science and Technology Center for Science of Information, under grant CCF-0939370.

\section{References}

[1] D. Avis, J. Hasegawa, Y. Kikuchi and Y. Sasaki, A quantum protocol to win the graph coloring game on all Hadamard graphs, Journal IEICE Transactions on Fundamentals of Electronics, Communications and Computer Sciences, Volume E89-A Issue 5, pp. 1378-1381, 2006.

[2] E. R. Barnes, A lower bound for the chromatic number of a graph, Contemporary Mathematics, 275 (2001), 3-12.

[3] R. Bhatia, Matrix analysis, Graduate text in mathematics, vol. 169, Springer

[4] Y. Bilu, Tales of Hoffman: Three extensions of Hoffman's bound on the graph chromatic number, J. Combin, Theory, Ser. B, 96 (2006), 608-613.

[5] B. Bollobás, The chromatic number of random graphs, Combinatorica 8(1), 49-55, 1988.

[6] B. Bollobás and V. Nikiforov, Cliques and the spectral radius, J. Combin. Theory Ser. B 97 (2007), 859-865. 
[7] A. Brouwer and W. Haemers, Spectra of graphs, Universitext, Springer, 2012; http://www.win.tue.nl/ aeb/2WF02/spectra.pdf

[8] P. J. Cameron, A. Montanaro, M. W. Newman, S. Severini, A. Winter, On the chromatic number of graph, The Electronic Journal of Combinatorics, 14, \#R81, 2007.

[9] D. Cvetkovic, Chromatic number and the spectrum of a graph, Publ. Inst. Math. (Beograd), 14(28) (1972), 25-38.

[10] D, Cvetkovic, P. Rowlinson, and S. Smic, An introduction to the theory of graph spectra, London Mathematical Society Student Texts 75, Cambridge University Press, 2010

[11] C. Edwards and C. Elphick, Lower bounds for the clique and the chromatic number of a graph, Discrete Appl. Math. 5 (1983) 51-64.

[12] C. Elphick, School Timetabling and Graph Colouring, PhD thesis (unpublished), University of Birmingham, UK, 1981.

[13] M. Gary and D. Johnson, Computers and Intractability: A guide to the theory of NP-completeness, Freeman 1978.

[14] C. D. Godsil and M. W. Newman, Colouring an orthogonality graph, SIAM Journal on Discrete Mathematics 22(2) (2008), pp.-683-692.

[15] C. Godsil, private correspondence, 2012.

[16] C. Godsil and G. Royle, Algebraic Graph Theory, Springer-Verlag, New York, 2001.

[17] G. Haynes, C. Park, A. Schaeffer, J. Webster, L. H. Mitchell, Orthogonal vector coloring, The Electronic Journal of Combinatorics, 17, \#R55, 2010; http://www . combinatorics.org/ojs/index.php/eljc/article/view/v17i1r55/

[18] W. Haemers, Eigenvalue Techniques in Design and Graph Theory, Mathematisch Centrum, Amsterdam, 1979.

[19] A. J. Hoffman, On eigenvalues and colourings of graphs, in: Graph Theory and its Applications, Academic Press, New York (1970), pp. 79-91.

[20] J. Hastad, Clique is hard to approximate within $n^{1-\varepsilon}$, Acta Math., 182:105-142, 1999.

[21] B. R. Myers and R. Liu, A lower bound for the chromatic number of a graph, Networks 1 (1972), 273-277.

[22] J. Jones, E. Knill, Efficient refocussing of one spin and two spin interactions for NMR quantum computation, J. Magn. Resonance 141 (1999), pp. 322-325

[23] D. Karger, R. Motwani and M. Sudan, Approximate graph coloring by semidefinite programming, Journal of the ACM 45(2), pp. 246-265, 1998.

[24] L. Lovász, On the Shannon capacity of a graph, IEEE Trans. Inf. Th., 25(1) (1979), $1-7$.

[25] V. Nikiforov, Some inequalities for the largest eigenvalue of a graph, Combin. Probab. Comput. 11 (2002), 179-189. 
[26] V. Nikiforov, Walks and the spectral radius of graphs, Linear Algebra Appl. 418, 257-268, 2006.

[27] V. Nikiforov, Chromatic number and spectral radius, Linear Algebra Appl. 426, 810$814,2007$.

[28] J. H. Smith, Some properties of the spectrum of a graph, pp. 403-406 in: Combinatorial Structures and their Applications, Proc. Conf. Calgary 1969, Gordon and Breach, New York, 1970.

[29] G. W. Stewart and Ji-guang Sun, Matrix perturbation theory, Academic Press, 1990.

[30] H. Wilf, Spectral bounds for the clique and independence numbers of graphs, J. Combin. Theory Ser. B 40(1986), 113-117.

[31] P. Wocjan, Computational power of Hamiltonians in quantum computing, Dissertation (unpublished), University of Karlsruhe, Germany, 2003;

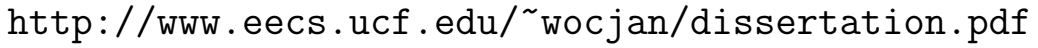

